\title{
Empirical methodology to determine inherent strains in additive manufacturing
}

\author{
Iñaki Setien $^{\mathrm{a}, *}$, Michele Chiumenti ${ }^{\mathrm{c}, \mathrm{d}}$, Sjoerd van der Veen ${ }^{\mathrm{b}}$, \\ Maria San Sebastian ${ }^{a}$, Fermín Garciandía a ${ }^{a}$ Alberto Echeverría ${ }^{a}$ \\ a IK4-LORTEK Technological Centre, Arranomendia kalea, 4A, 20240 Ordizia, Spain \\ b AIRBUS Structures Research and Integration, ESIRNM, 18 Rue Marius Terce, 31300 Toulouse, France \\ c Centre Internacional de Mètodes Numèrics en Enginyeria (CIMNE), Building C1, Campus Nord UPC, Gran Capitán S/N, E-08034 \\ Barcelona, Spain \\ ${ }^{\mathrm{d}}$ Universitat Politècnica de Catalunya, Jordi Girona 1-3, Edifici C1, E-08034 Barcelona, Spain
}

\section{A R T I C L E I N F O}

\section{Article history:}

Available online $\mathrm{xxxx}$

\section{Keywords:}

Additive manufacturing

Inherent strain

Finite element modelling

\begin{abstract}
A B S T R A C T
Part distortion is a critical issue during Additive Manufacturing (AM) of metallic parts since it prevents this technology from being implemented at industrial level. To this regard, distortion prediction even from design stage has become crucial. Actually, numerical modelling methodologies play an important role here. Different modelling approaches have been developed but one of the most computationally efficient methodology to predict distortion is the so called inherent strain method. In this work an empirical methodology to determine inherent strains is presented. This is the input data in simplified Finite Element (FE) models in order to predict distortion and residual stress fields. These inherent strains are calculated considering layer lumping strategies that might be adopted in the numerical model as well. The procedure has been developed and validated using the wellknown twin-cantilever beam structure. Ti-6Al-4V beams have been manufactured by LPBF technology following different scanning strategies. Distortion after support removal has been measured in order to be compared against numerical results. The methodology has been applied at coupon level giving accurate results and providing a preliminary validation.
\end{abstract} (C) 2018 Elsevier Ltd. All rights reserved.

\section{Introduction}

Additive Manufacturing (AM) gathers various techniques for building three dimensional objects in a layer by layer sequence starting from a digital model [1,2]. This incomparable characteristic allows the production of complex, customised and fully optimised parts directly from the design stage, avoiding the need of expensive and restrictive conventional manufacturing processes. Therefore, it is nowadays widely considered that AM is the new paradigm for design and production of high performance components.

Laser Powder Bed Fusion (LPBF) is one of the most widespread powder-bed technology for metallic components. In these processes, the material is locally and rapidly heated and then allowed to solidify in order to form a dense geometry. The temperature history experienced by the material determines the microstructure and therefore the mechanical properties. Large thermal strains are involved in the process, generating residual stresses and distortions [3], affecting significantly the mechanical properties and causing dimensional inaccuracies to the final part. Henceforth, there are still serious obstacles toward its wider acceptance and establishment into modern industrial practice.

\footnotetext{
* Corresponding author.

E-mail address: isetien@lortek.es (I. Setien).
} 
The issues above are unknowns for the designer each time a part is manufactured by AM. Currently, they are tackled by expensive trial and error experiments until all requirements are fulfilled. This implies high manufacturing costs, increased even more due to the waste and scrap generated when quality inaccuracies between the designed and built components occur. Therefore, predictive methods such as the computational simulation are essential in an attempt to predict and control the aforementioned phenomena even at the design stage. Thermo-mechanical and thermo-microstructure behaviour modelling of AM processes is increasingly useful and necessary to deal with these challenges efficiently [4].

AM process modelling has its own challenges due to the multiphysics and multiscale character of the process. Its physical fundamentals are very similar to those of multi-pass welding processes. Thus, Finite Element Method (FEM) based on welding modelling techniques developed so far, can be adapted to develop AM simulations [5-8]. Accurate coupled thermomechanical simulations are considered as high-fidelity numerical models where the real weld pool moving along the scan path is modelled in detail according to Computational Welding Mechanics (CWM). Many researchers have successfully applied and validated this method in order to predict thermal, mechanical and microstructural histories [9-16]. Nevertheless, these high-fidelity modelling approaches have only been applied at coupon level since they are computationally impracticable for real size parts. Added difficulties such as longer process time, higher number of time-steps, etc. are the reasons behind this limitation. Particularly, AM processes like LPBF, where the weld pool size is very small compared to part size, are affected by these issues.

Consequently, several simplified methodologies have been developed in order to model this process at industrial scale level with the main aim of predicting distortions and residual stresses [17-21].

These methods can be classified into two categories. On the one hand, there are methods focused on reducing the computational effort by making more efficient and scalable the algorithms and the numerical methods required to solve the Finite Element (FE) problem [22], or by reducing the model size applying adaptive meshing techniques [23].

On the other hand, there are engineering approaches which make assumptions to simplify the physics behind the metal deposition process with the purpose of simulating distortions and/or residual stresses at part level. Some researchers [9] have simplified the heat source modelling, i.e. applying equivalent energy input at hatch, layer or even layer-compound level. Others have avoided even the heat transfer problem through applying equivalent shrinkage load in a purely mechanical analysis $[21,24,25]$. One of the most promising and efficient approach is the inherent strain method $[25,26]$. This wellknown methodology has already been successfully applied to both CWM and AM processes [19]. It allows for replacing computationally costly thermo-mechanical simulations by a linear-elastic-mechanical one. The key point in this simplified analysis is to define which is the inherent strain or shrinkage load to apply in the model in order to capture the mechanical response of the fabricated component.

There are two approaches to determine these inherent strains as proposed in CWM [27]: (a) Multi-scale or local-global modelling; (b) Empirical approach.

The multi-scale modelling option consists of solving micro and/or meso-scale models to determine inherent strains at local level whereas in the second option, they are calibrated by means of experimental tests and calculations.

The work presented in this paper is focused on the development of an empirical methodology to determine inherent strains. In Section 2 all the experimental work carried out to develop the methodology and its validation is explained. The FE model based on the inherent strain methodology to predict distortions and residual stresses fields in LPBF is described in Section 3. The model is then calibrated looking for the most suitable inherent strains to be used. Section 4 is dedicated to the detailed description of the empirical procedure to determine inherent strains. Section 5 gathers both experimental and numerical results achieved for both calibration and validation stages. Finally, Section 6 presents the main conclusions of this work.

\section{Experimental work}

An overview of the experimental work carried out is provided here.

\subsection{Experimental set-up}

Several experimental trials were conducted in order to obtain data for the calibration phase described in Section 4. Different coupons were manufactured using a LPBF process. A SLM 250 (from MCP-Realizer) machine equipped with $200 \mathrm{~W}$ fibre laser was used. The machine has a construction space of $250 \mathrm{~mm} \times 250 \mathrm{~mm} \times 300 \mathrm{~mm}$ which can accommodate the production of components with maximum size. It must also be noted that the machine works with a protective atmosphere, being $0.2 \%$ the maximum oxygen allowable, in order to prevent titanium parts from oxidation.

Twin-cantilever type structures made of Ti-6Al-4V were fabricated over several thick baseplates $(150 \mathrm{~mm} \times 150 \mathrm{~mm} \times$ $15 \mathrm{~mm})$. Fig. 1 shows the geometry of the sample and its dimensions.

As shown in Fig. 2, 3 different scanning strategies with respect to the baseplate's reference system $(x y)$ were employed to print the twin-cantilevers. A total number of 11 twin-cantilevers were manufactured in 4 baseplates and different batches. Their distribution and the scanning strategy assigned to each baseplate is represented in Fig. 3. The orientation of twincantilevers with respect to the main reference system $(x y)$ leads to 5 different scanning strategies (see Fig. 4). The local coordinate system $x_{1} y_{1}$ attached to the beam is shown in Fig. 3. Hence, on the one hand 3 sets of twin-cantilevers were manufactured with longitudinal, transversal and $45^{\circ}$ stripes without any rotation between layers. On the other hand, 2 more 


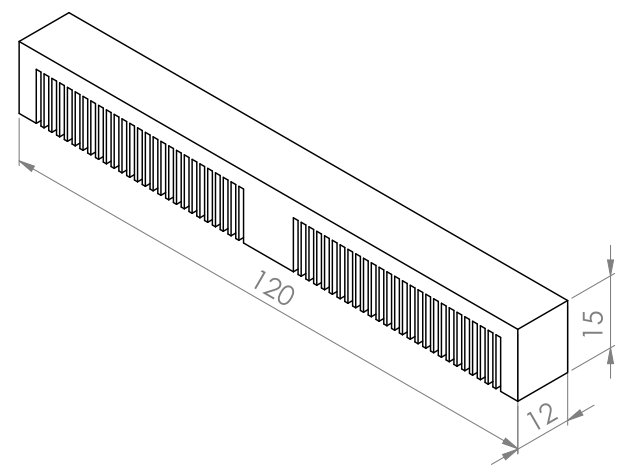

Fig. 1. Twin-cantilever specimen with dimensions in $\mathrm{mm}$.

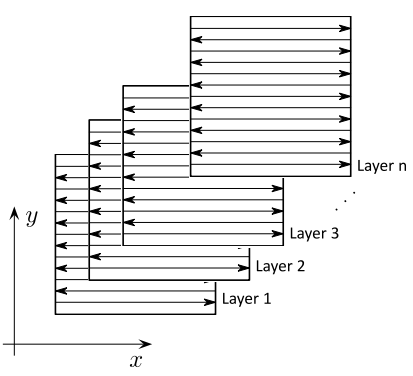

(a)

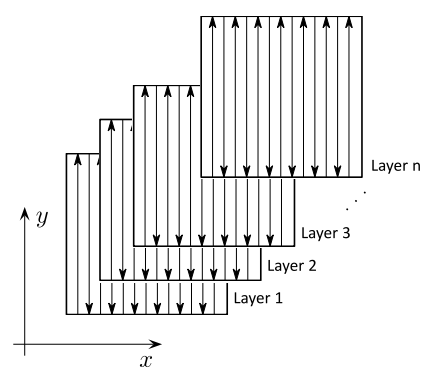

(b)

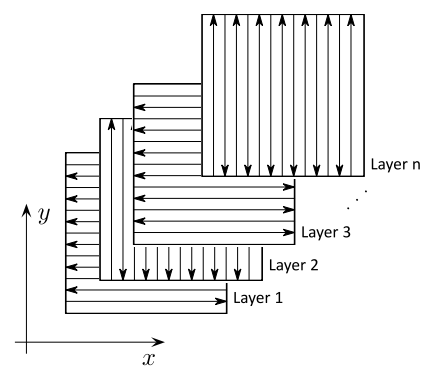

(c)

Fig. 2. Considered scanning strategies taking as reference the baseplate. (2(a)) $0^{\circ}$ line constant scan pattern. (2(b)) $90^{\circ}$ line constant scan pattern. $(2(\mathrm{c}))$ $90^{\circ}$ rotating scan pattern.

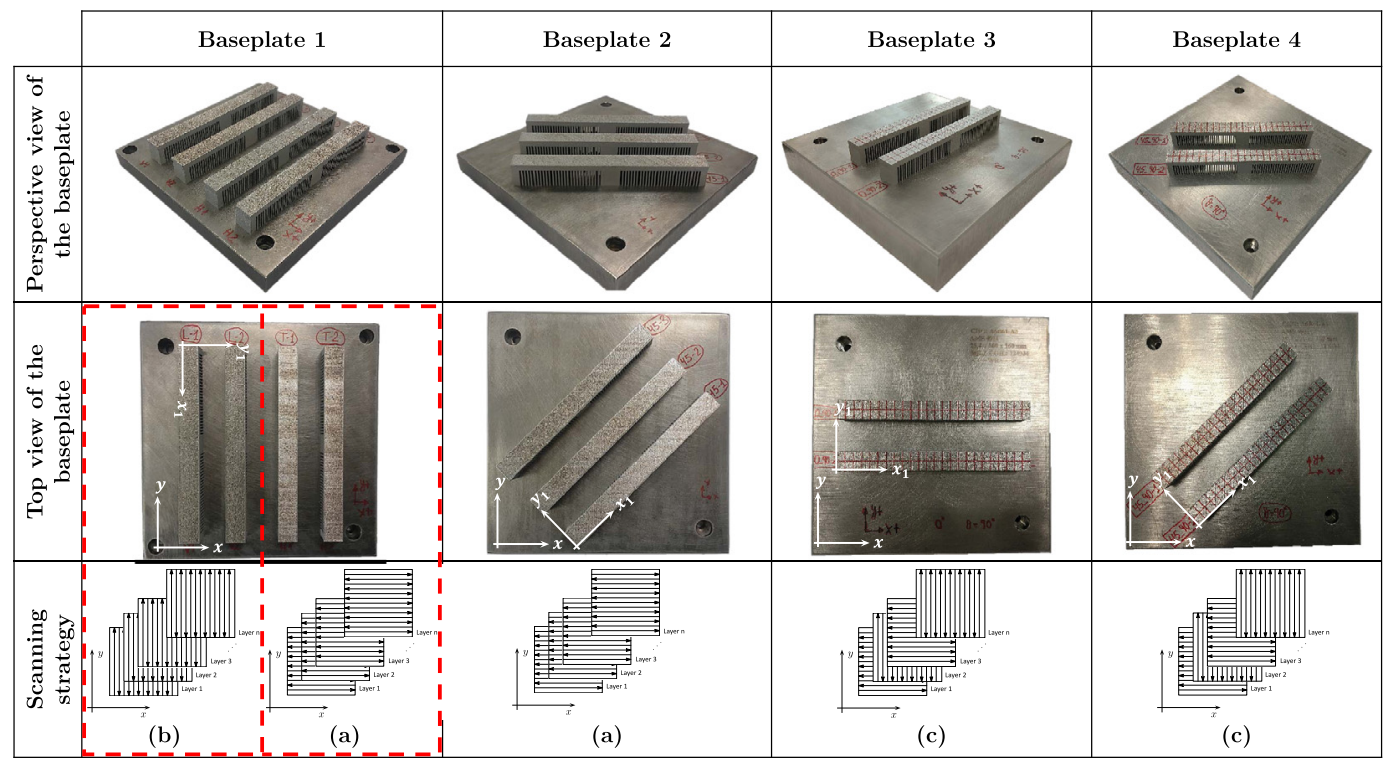

Fig. 3. Manufactured baseplates and corresponding scanning strategies used in each baseplate.

sets of beams were manufactured with $90^{\circ}$ rotating scan pattern with different initial offset of the rotation angle $\left(0^{\circ}\right.$ and $\left.45^{\circ}\right)$. 


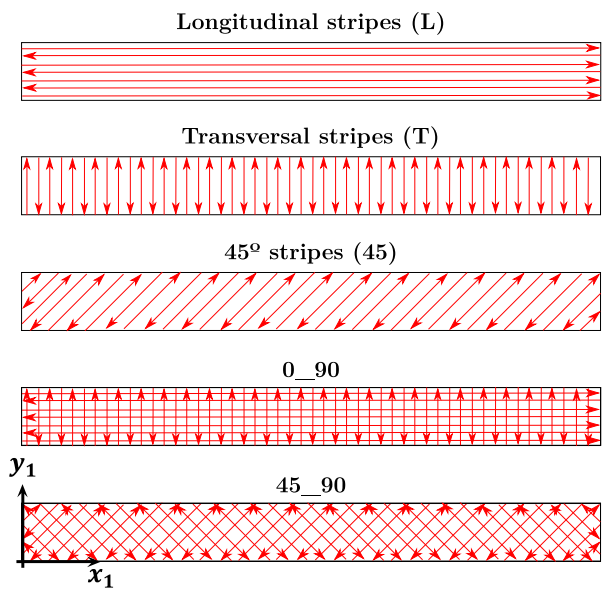

Fig. 4. Scanning strategies summary taking as reference the local coordinate system of the beam.

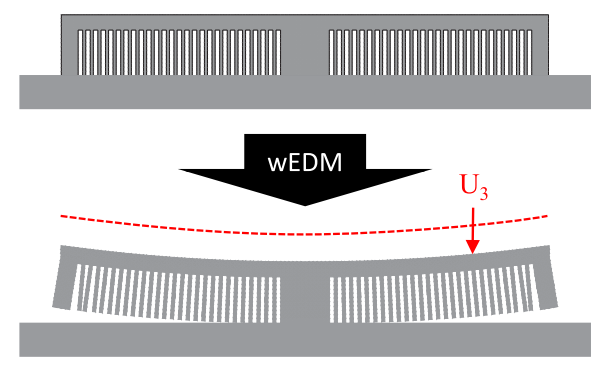

Fig. 5. Twin-cantilever cutting and vertical distortion measurement.

Table 1

LPBF process parameters.

\begin{tabular}{llll}
\hline & Hatch & Boundary & Contour \\
\hline Laser power $(\mathrm{W})$ & 200 & 200 & 200 \\
Scanning speed $(\mathrm{mm} / \mathrm{s})$ & 950 & 1100 & 1100 \\
Hatch distance $(\mu \mathrm{m})$ & 120 & - & - \\
Beam diameter $(\mu \mathrm{m})$ & 180 & 180 & 180 \\
\hline
\end{tabular}

The use of optimum process parameters is extremely important for producing satisfactory parts using these processes. In our particular case, previously optimised Ti-6Al-4V process parameters were employed (see Table 1 ). These parameters were adjusted for hatch, boundary and contour zones. The preheating temperature was $200{ }^{\circ} \mathrm{C}$ and the nominal layer thickness was $50 \mu \mathrm{m}$.

\subsection{Measurement equipments}

After manufacturing and without any stress relaxation treatment, the specimens were partially cut from the baseplate by wire Electrical Discharge Machining (wEDM), leaving the middle column attached to the baseplate. Thus, residual stress field cumulated during the manufacturing process was redistributed leading to the bending of the part. The final vertical distortion was measured on the top surface centre-line along the $x_{1}$ direction as illustrated in Fig. 5. Measurements were carried out by means of a Coordinate-Measuring Machine (CMM) every $5 \mathrm{~mm}$ along the centre-line in $x_{1}$ direction. This bending deformation is an indirect way of assessing the magnitude of residual stresses. Therefore, it has been used for the calibration of the inherent strains.

\section{Finite element modelling of AM process by LPBF}

\subsection{Inherent strain method}

The inherent strain method is an efficient method which enables a fast prediction of the residual stresses and distortions. The origin of the inherent strain method is directly related to Computational Welding Mechanics and it has been widely 


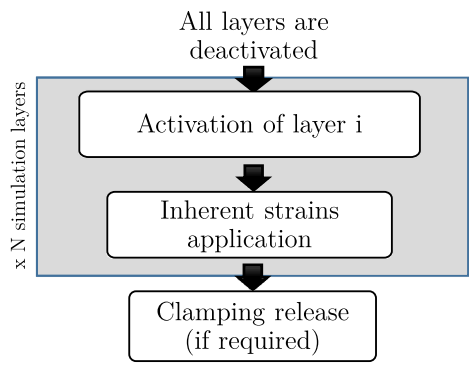

Fig. 6. Simulation steps required by the inherent strain based simplified model.

used [25]. Briefly, it consists of an elastic FE quasi-static analysis where the deformation is induced by user-defined inherent strains. This approach is very compelling from a computational point of view since time-consuming thermo-mechanical simulations are avoided.

During the heating and cooling cycles of the AM process, the total strains $\boldsymbol{\varepsilon}^{\text {total }}$ can be split into elastic strains $\boldsymbol{\varepsilon}^{\text {elastic }}$, thermal strains $\boldsymbol{\varepsilon}^{\text {thermal }}$, plastic strains $\boldsymbol{\varepsilon}^{\text {plastic }}$, strains induced by the phase transformation $\boldsymbol{\varepsilon}^{\text {phase }}$ and creep strains $\boldsymbol{\varepsilon}^{\text {creep }}$ [28]. Hence:

$$
\varepsilon^{\text {total }}=\varepsilon^{\text {elastic }}+\varepsilon^{\text {thermal }}+\varepsilon^{\text {plastic }}+\varepsilon^{\text {phase }}+\boldsymbol{\varepsilon}^{\text {creep }}
$$

The inherent strains $\boldsymbol{\varepsilon}^{\text {inh }}$ are defined as:

$$
\varepsilon^{\text {inh }}=\varepsilon^{\text {total }}-\varepsilon^{\text {elastic }}=\varepsilon^{\text {thermal }}+\varepsilon^{\text {plastic }}+\varepsilon^{\text {phase }}+\varepsilon^{\text {creep }}
$$

The balance of momentum equation for a quasi-static analysis is:

$$
\nabla \cdot \sigma+\boldsymbol{b}=\mathbf{0}
$$

where $\boldsymbol{\sigma}$ is the stress tensor and $\boldsymbol{b}$ are the body forces.

The stresses can be calculated by:

$$
\sigma=\boldsymbol{D}^{e} \varepsilon^{\text {elastic }}=\boldsymbol{D}^{e}\left(\boldsymbol{\varepsilon}^{\text {total }}-\boldsymbol{\varepsilon}^{\text {inh }}\right)
$$

where $\boldsymbol{D}^{e}$ is the elastic constitutive tensor.

The inherent strain method has been successfully adapted to Powder Bed Fusion (PBF) processes in a multi-scale simulation framework [19]. Typically, the building strategy is approximated by layer-by-layer sequence. Hence, at each time-step a new layer is added to the computational domain. In fact, it is a common practice to discretise a part into layers with a thickness which is multiple of the real layer thickness referred to as lumping strategy.

Hence, according to the definition of the inherent strain tensor components, it is possible to take into account the "effects" of the actual scanning strategy.

Material deposition modelling must also be addressed in this methodology. From the numerical point of view, this implies the use of a FE activation strategy. At each time step a new layer must be activated and the AM building process is simulated as a sequence of mechanical calculations, one for each manufacturing layer.

The layer activation strategy is based on the so called "born and death elements" technique. According to this activation strategy, elements are not part of the model until they are activated according to the nominal height due to the powder feeding in PBF processes $[15,16,29]$.

Thus, this simplified model consists of a mechanical linear elastic problem complemented with the activation strategy and user defined inherent strains. As a result, the whole analysis requires several simulation steps which are summarised in the flowchart depicted in Fig. 6. Initially, the slicing of the mesh is defined automatically according to the layer thickness and orientation defined in the input data. In the first step, all layers are removed from the computational domain. A new layer is added in each step together with the corresponding inherent strains. Finally, the boundary conditions corresponding to the clamping on the base-plate can be removed (cutting stage). Fig. 7 shows an intermediate state of a simulation where the building evolution can be observed. The key factor in this simplified methodology is how inherent strains are determined considering also the effect of the layer lumping. This is detailed in Section 4.

\subsection{Finite element modelling}

A 3D purely mechanical model was developed using the commercial software ABAQUS to simulate the deformation of the twin-cantilever beams under different scanning strategies. The modelling methodology and particularly the layer lumping enables to use a relatively coarse FE mesh. It consists of a structured cartesian mesh of 15,552 hexahedral elements 


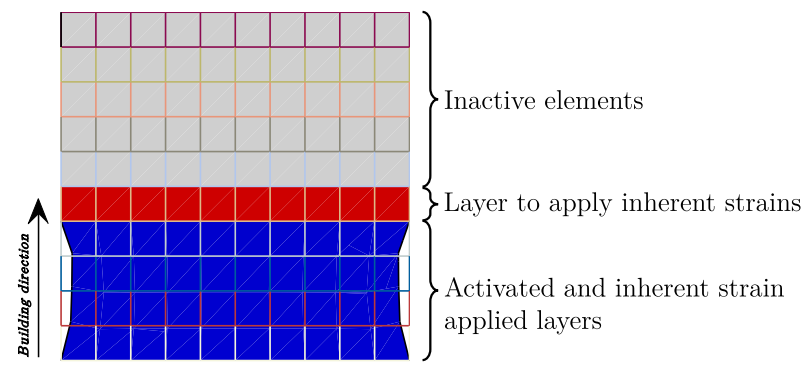

Fig. 7. Intermediate state of the simulation based on inherent strain methodology.

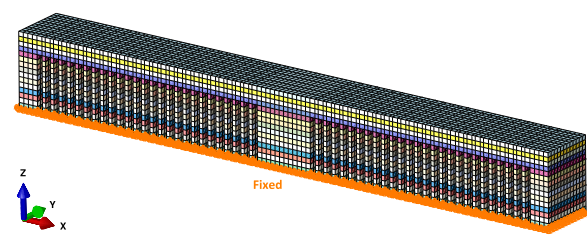

(a) Boundary conditions during printing.

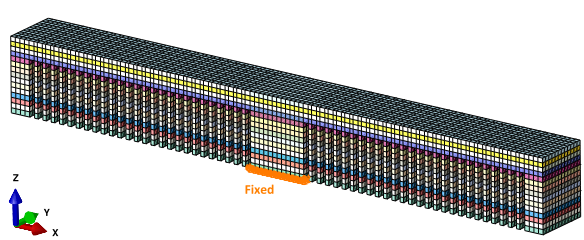

(b) Boundary conditions when the supports are removed.

Fig. 8. Twin-cantilever finite element mesh and boundary conditions.

and 26,416 nodes. Moreover, symmetry boundary conditions were used when the scanning strategy presents symmetry, reducing the model size to a quarter.

Fig. 8 shows the entire mesh. Each simulation layer, which can be identified in the figure by the different colours, is $1 \mathrm{~mm}$ thick. The lumping ratio was 20:1. Thus, 20 real layers were represented by 1 simulation layer. Moreover, each layer was discretised by 1 element through the building direction.

The building platform was not considered in the model since it was represented by a fully constrained bottom region (Fig. 8(a)). Moreover, supports removal by wEDM was modelled as a simple change of boundary conditions (Fig. 8(b)). After the wEDM, only the bottom surface of the middle column was fixed, releasing all clamping belonging to the supporting structures.

The Ti-6Al-4V material properties used to characterise the constitutive tensor are: Young modulus $(E=113,340 \mathrm{MPa})$ and Poisson ratio $(v=0.3)$. The wall-clock time required, exploiting 12 Intel Xeon E5 cores, was about $20 \mathrm{~s}$.

\section{Inherent strains calibration}

As pointed out in Section 3.1, the key factor at the methodology is to characterise the prescribed deformations responsible for the formation of both residual stresses and the distortions (after the stress release by cutting operation).

Two methods can be distinguished for this purpose:

- Reduced order methods.

- Empirical methods.

\subsection{Reduced order methods}

This multiscale modelling approach proposed by different authors $[19,20,30]$ is a promising and fully predictive way of determining the inherent strain tensor with a reduced dependency from experimental data. It consists of an hierarchical procedure whereby inherent strains are predetermined through high-fidelity FE models. A coupled thermo-mechanical analysis is carried out considering the actual scanning strategy sequence by a moving heat source representing the power input. As a result, both thermal and visco-plastic deformations can be accurately computed. Hence, the corresponding inherent strains can be identified. Note that this procedure can be repeated at local level for different scanning strategies so that an off-line database of inherent strains can be constructed according to different process parameters. Later, this input can be used for the actual parts (global parts). Hence, a local-global analysis is carried out (see Fig. 9).

However, the definition of representative local models able to take into account all the fundamental process parameters is not straightforward. Neither the model set-up nor the mapping between high-fidelity local and global analysis are not completely clear yet. Henceforth, we do not choose this option for determining inherent strains. 


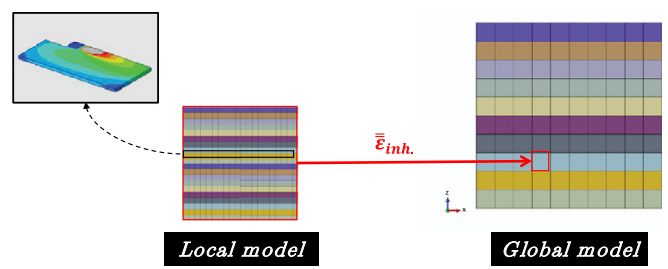

Fig. 9. Multiscale simulation based inherent strain determination scheme.

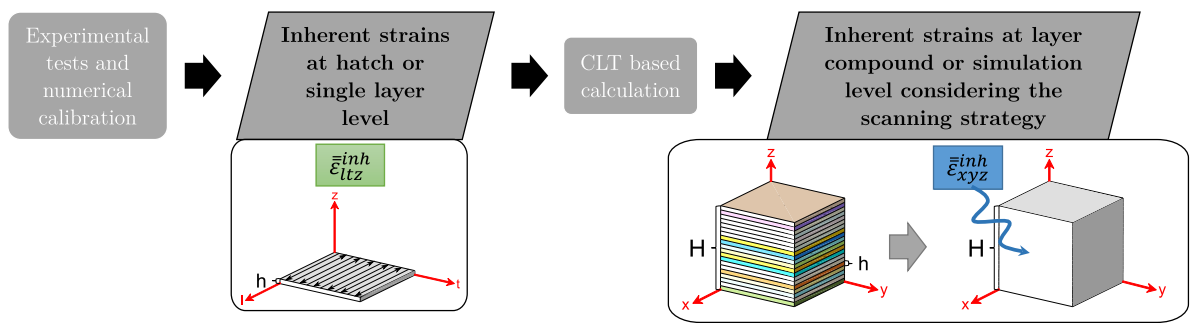

Fig. 10. Inherent strain determination strategy based on experimental tests.

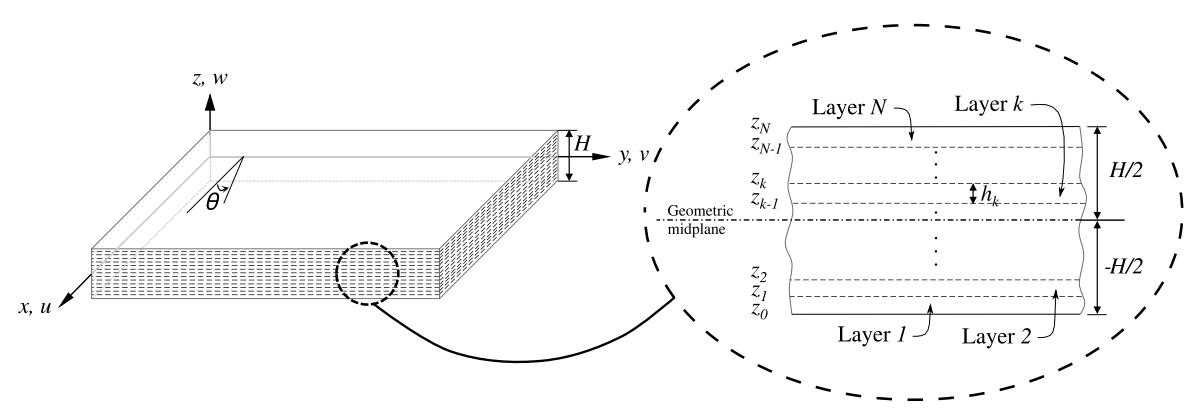

Fig. 11. Schematic representation of layers.

\subsection{Empirical method}

The empirical strategy adopted to obtain the inherent strains is presented in the flowchart shown in Fig. 10. According to this flowchart, the characteristic inherent strains which belong to each single layer are obtained by experimental tests and by iterative fitting. In this regard, according to the layer thickness used in the simulation, the equivalent inherent strain is calculated. This methodology is very similar to the one used for Classical Laminate Theory (CLT) to characterise the behaviour of composite laminates [31,32]. In fact, the layer-by-layer manufacturing using PBF technology has many similarities with the technology used to produce composite laminates. In both cases, the final structure is made layer-wise and it exists an induced anisotropy. In AM, the hatching sequence generates the same effects as due to fibres orientation.

The following definitions are necessary:

- It is assumed that the layer thickness used for the simulation $(\mathrm{H})$ is composed by $\mathrm{N}$ deposited layers, being $\mathrm{h}$ the thickness of each one of them (see Fig. 11).

- $(l, t, z)$ is the local coordinate system, following the orientation of the local hatching defined for each layer.

- $(x, y, z)$ is the global coordinate system, attached to the building platform and used to define the hatches' orientation for each layer (see Fig. 11).

- The scanning strategy can be represented using the same nomenclature as for the stacking sequence of composite laminates [31]. Therefore, the orientation of hatches is established for each layer by defining the rotation angle $(\theta)$, with respect to the reference $x$-axis (see Fig. 11). (i.e. a scanning strategy of $90^{\circ}$ is represented as $[0 / 90]_{N}$ ). 


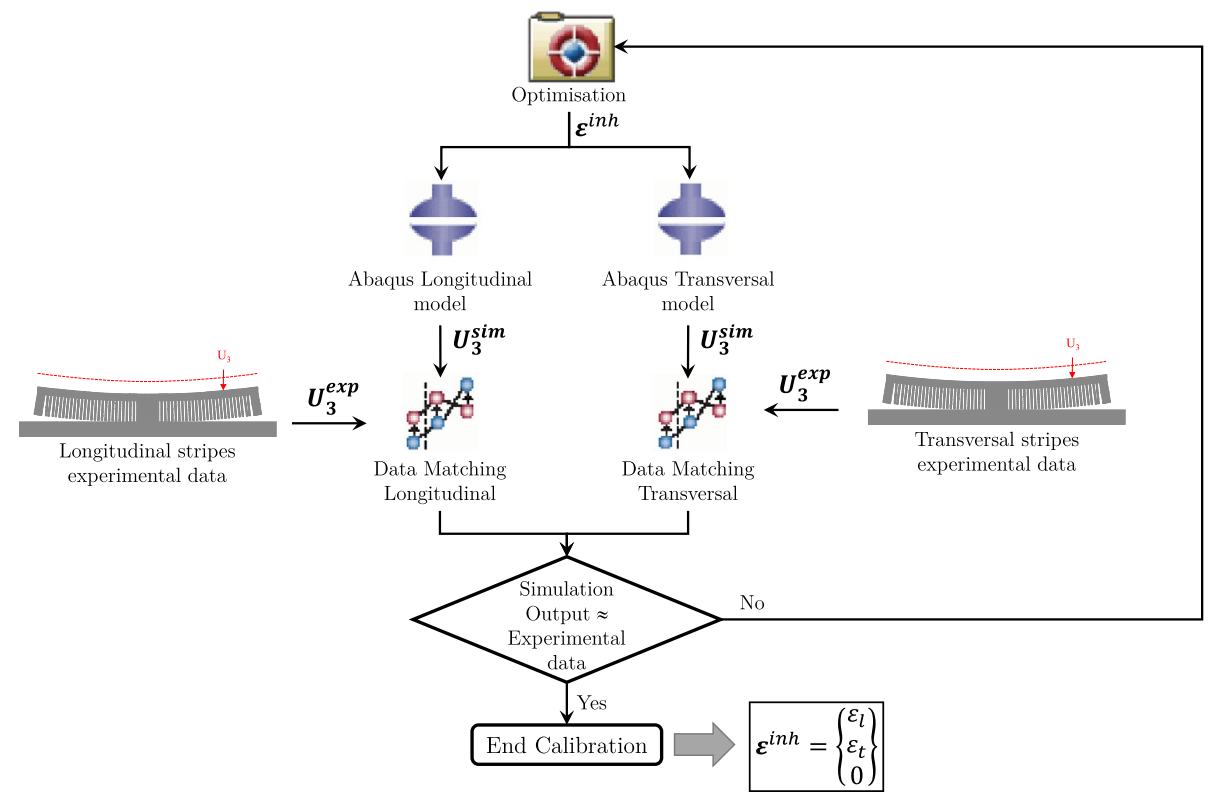

Fig. 12. Isight data matching optimisation loop for characteristic inherent strain calibration.

\subsubsection{Inherent strains at hatch or single layer level}

In order to determine the inherent strains at layer level, the following two basic hypotheses are made:

- The inherent strain tensor is orthogonal due to the actual nature of hatches.

- The inherent strain component in the building direction is zero. Hence, only in-plane components are taken into account. This hypothesis assumes that the vertical shrinkage of the deposited layer is compensated by the recoating phase of the PBF process. Therefore, from the modelling point of view, the inherent strain component in the building direction can be neglected.

The iterative calibration process employed is presented in Fig. 12. The residual stresses are proportional to the inherent strains applied to the structure. Therefore, an indirect measurement of this residual stress state enables to calculate the inherent strain field through inverse analysis. In our particular case, the twin-cantilever beam geometry (see Fig. 1) is proposed since it allows for a simple indirect measurement of the residual stresses as a function of its bending deformation after the cutting phase.

According to the aforementioned hypothesis, two inherent strain components must be determined only. Thus, at least two experiments are required. Taking advantage of the orthogonal nature of hatches, two orthogonal scanning strategies are set-up for the same twin-cantilever beam: longitudinal and transversal hatching as depicted in Fig. 4 . Thus, 2 different residual stress state and consequently 2 different final bending deformation are obtained. An additional scanning strategy, with a orientation of $45^{\circ}$, is used to validate the model.

The relationship between inherent strains and distortion is determined through an optimisation loop where the objective function is the difference between the numerical results and the experimental measurements.

The optimisation problem has been solved via Isight commercial framework, combining: Abaqus FE model, data matching algorithm and experimental data input (see the flowchart in Fig. 12). Hooke-Jeeves direct penalty technique is used to solve this optimisation problem.

As a result, the inherent strain tensor at layer level is obtained and then used as a reference data for similar scanning strategies. It must be noted that the obtained inherent strain tensor is dependent on the process parameters used to manufacture the twin-cantilever beam.

Moreover, a simple verification of calibrated results is done by applying the obtained inherent strain tensor to $45^{\circ}$ scanning strategy and comparing the numerical results against the experimental data.

\subsubsection{Inherent strains for the numerical model}

Although inherent strains can be obtained at layer level, some extra hypotheses are needed to account for the layer lumping used in the model. According to Kirchhoff hypothesis (CLT theory), strains vary linearly through the thickness of 
the laminate structure as:

$$
\left\{\begin{array}{l}
\varepsilon_{x} \\
\varepsilon_{y} \\
\varepsilon_{z}
\end{array}\right\}=\left\{\begin{array}{c}
\varepsilon_{x}^{0} \\
\varepsilon_{y}^{0} \\
\gamma_{x y}^{0}
\end{array}\right\}+z\left\{\begin{array}{l}
\kappa_{x} \\
\kappa_{y} \\
\kappa_{z}
\end{array}\right\}=\left\{\varepsilon^{0}\right\}+z\{\kappa\}
$$

where $\varepsilon_{x}^{0}, \varepsilon_{y}^{0}, \varepsilon_{z}^{0}$ are the midsurface strains and $\kappa_{x}, \kappa_{y}, \kappa_{z}$ are the curvatures due to bending and twisting.

An analogous hypothesis can be used for AM processes where the equivalent inherent strains can be calculated by Eqs. (6) and (7), summing the inherent strains of all the layers defining the lumped structure:

$$
\begin{gathered}
\boldsymbol{\varepsilon}^{0 I n h}=\frac{1}{N^{2}} \sum_{k=1}^{N}\left\{\begin{array}{c}
\varepsilon_{x}^{I n h} \\
\varepsilon_{y}^{I n h} \\
\varepsilon_{x y}^{I n h}
\end{array}\right\}_{k}(4 N-6 k+3)=\frac{1}{N^{2}} \sum_{k=1}^{N} \varepsilon_{k}^{I n h}(4 N-6 k+3) \\
\boldsymbol{\kappa}^{I n h}=\frac{-6}{h N^{3}} \sum_{k=1}^{N}\left\{\begin{array}{c}
\varepsilon_{x}^{I n h} \\
\varepsilon_{y}^{I n h} \\
\varepsilon_{x y}^{I n h}
\end{array}\right\}_{k}(N-2 k+1)=\frac{-6}{h N^{3}} \sum_{k=1}^{N} \varepsilon_{k}^{I n h}(N-2 k+1)
\end{gathered}
$$

where $N$ is total number of lumped layers and $\varepsilon_{k}^{I n h}$ is the characteristic inherent strain at layer $k$ (see Fig. 12).

Both expressions are general. In case the scanning strategy presents a periodic rotation angle, the equivalent inherent strain of the lumped structure reduces to:

$$
\boldsymbol{\varepsilon}_{\text {periodic }}^{\text {OInh }}=\frac{\varepsilon_{l}+\varepsilon_{t}}{2}\left\{\begin{array}{l}
1 \\
1 \\
0
\end{array}\right\}
$$

where $\varepsilon_{l}$ and $\varepsilon_{t}$ are the longitudinal and transversal components of inherent strains calibrated at layer level.

Interestingly, periodic scanning strategies result in in-plane isotropic inherent strains which do not depend on the rotation angle as long as periodicity is fulfilled. Consequently, the application of such inherent strain load is straightforward since the material orientation definition is not needed. In order to be periodic, the rotation angle must satisfy the condition rule in Eq. (9). Furthermore, the layer thickness to be considered in the simulation is determined by Eq. (10).

$$
\begin{aligned}
& \frac{\pi}{\theta} \in \mathbb{N} ;(\theta<\pi) \\
& \exists n \in \mathbb{N}, H=n \frac{\pi}{\theta} h
\end{aligned}
$$

\section{Results and discussion}

\subsection{Experimental results}

The final manufacturing of all twin-cantilevers fabricated following the procedure explained in Section 2 is shown in Fig. 13. The front view provided for each type of twin-cantilever reveals the effect of the chosen scanning strategy.

As shown in Fig. 3, two twin-cantilevers were fabricated for each scanning strategy except for $45^{\circ}$ case, where one extra sample was manufactured. These repetitions enable a good experimental error estimation. Moreover, each twin-cantilever can be seen as two simple cantilever beams. Therefore, distortion data gathered from each structure is double (see Fig. 14) allowing for a better error distribution. All distortion curves are represented along the longitudinal axis [0 $\leq x \leq 60 \mathrm{~mm}$ ].

Distortion results for all types of cantilevers according to the scanning strategies defined in Fig. 4 are plotted in Fig. 15. In both graphs the vertical distortion results is normalised with respect to the longitudinal scanning. A clear influence of the scanning strategy can be noticed in the final distortion. Longitudinal stripes lead to the largest distortions whereas transversal stripes to the less pronounced ones. It is also remarkable the fact that the magnitude of the distortion corresponding to $45^{\circ}$ stripes is the average of the longitudinal and transversal distortions respectively. Observe that the scanning patterns defined with a periodic rotation with respect to the reference system, show very similar results as depicted in Fig. 15(b). This result is in accordance with Eq. (8) which states that scanning strategies with periodic rotation lead to in-plane isotropic inherent strains.

In addition, the aforementioned curves are also very similar to the results obtained for the $45^{\circ}$ stripes case.

\subsection{Calibrated inherent strains}

The calibration of the characteristic inherent strains was conducted following the methodology described in Section 4.2.1. The normalised inherent strain tensor obtained after the iterative data matching, can be observed in Eq. (11). The normalisation of the strain tensor was done with respect to its longitudinal component. 


\section{ARTICLE IN PRESS}
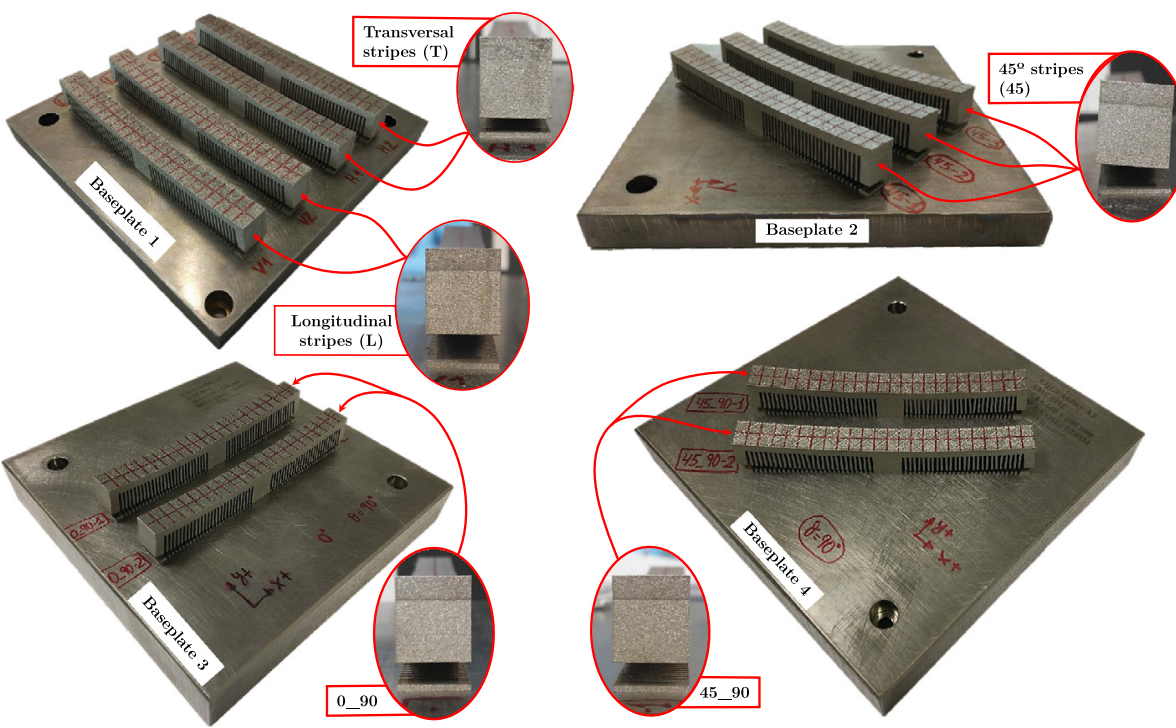

Fig. 13. Final state (after support removal) of all manufactured twin-cantilever beams.

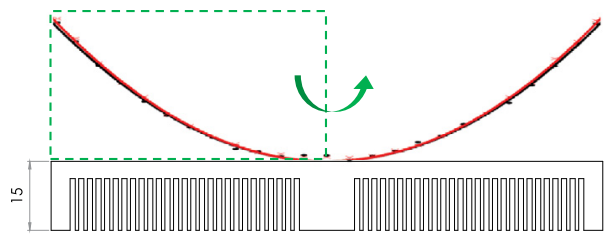

Fig. 14. Distortion data reflected with respect to the middle length of the twin-cantilever.

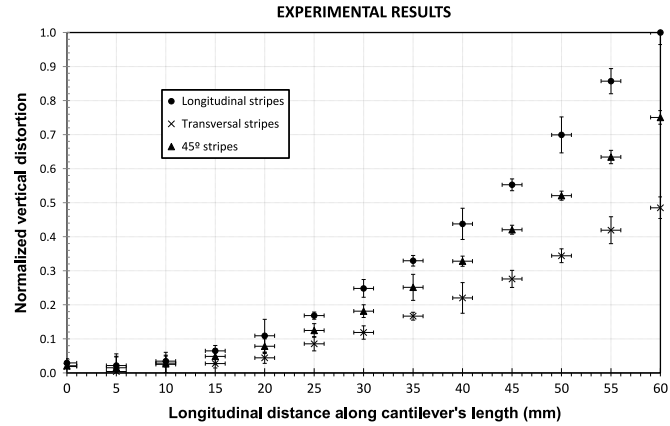

(a)

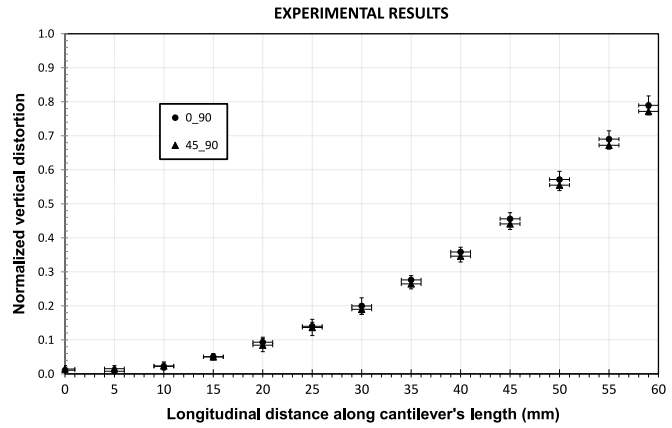

(b)

Fig. 15. Distortion measurement results along the top surface for all twin-cantilevers. (15(a)) No rotation between layers. (15(b)) $90^{\circ}$ rotation between layers.

Around 50 iterations were performed to fit numerical results with experimental data until the sum of the squared difference was lower than $10 \%$. To this regard, Figs. 16(a)-16(c) show the quality of the fitting carried out.

The vertical distortion obtained by FEM after applying the calibrated inherent strain can be observed in Figs. 17(a)-17(c). The deformed shape of twin-cantilevers is also in agreement with experimental results. It is remarkable how the numerical model is able to predict non-symmetrical deformations of the $45^{\circ}$ stripes twin-cantilever (see Fig. 17(c)).

$$
\boldsymbol{\varepsilon}^{i n h}=\left\{\begin{array}{c}
\varepsilon_{l} \\
\varepsilon_{t} \\
0
\end{array}\right\}_{l t z}=\left\{\begin{array}{c}
-1 \\
-0.33 \\
0
\end{array}\right\}
$$




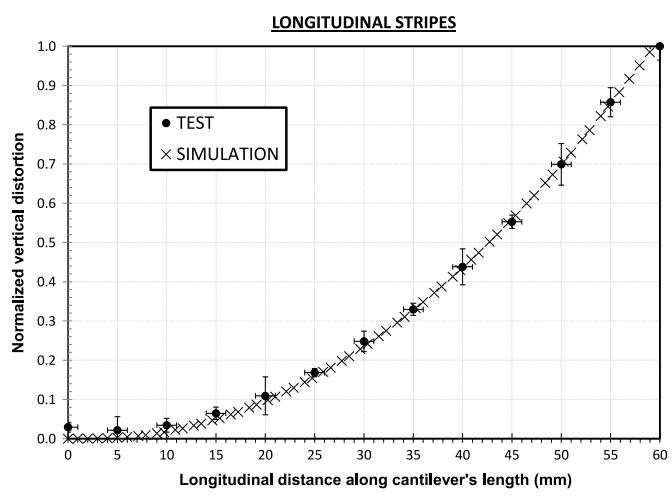

(a) Longitudinal stripes twin-cantilever.

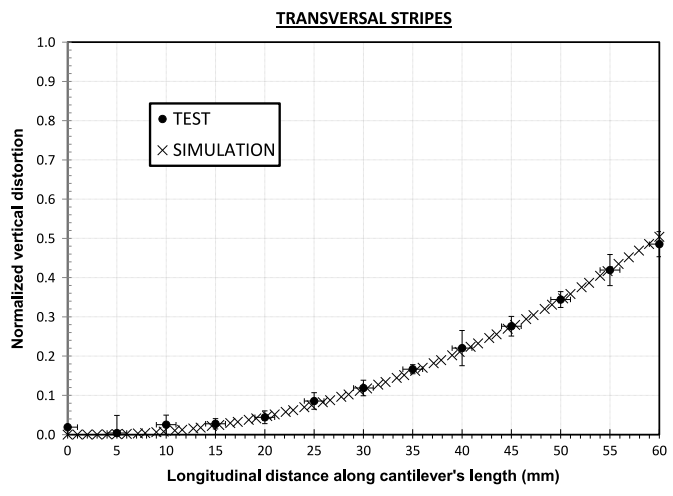

(b) Transversal stripes twin-cantilever.

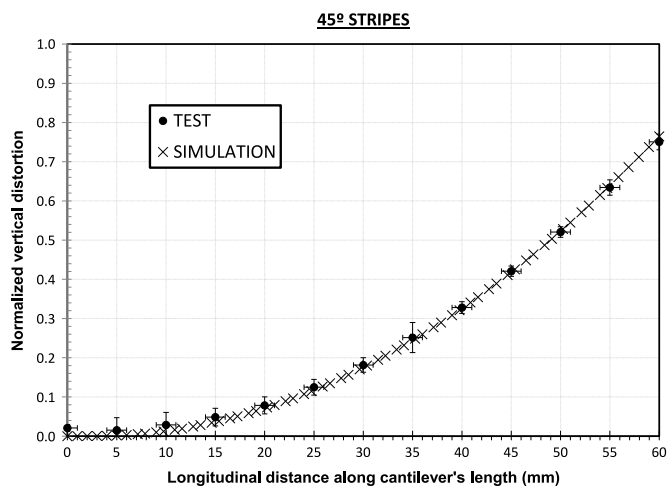

(c) $45^{\circ}$ stripes twin-cantilever.

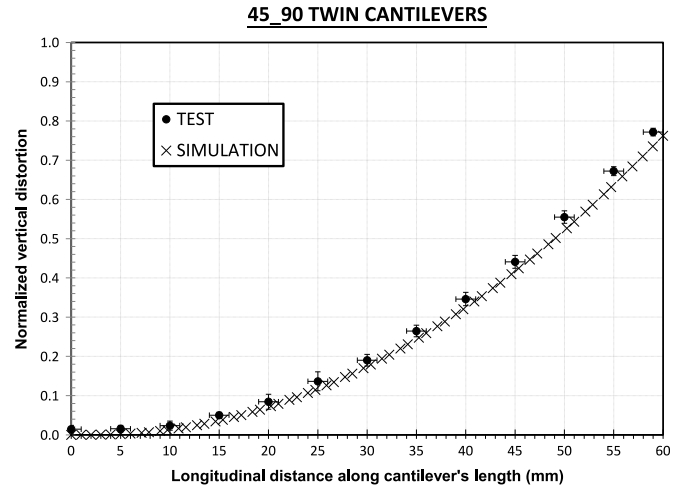

(d) 0_90 twin-cantilever.

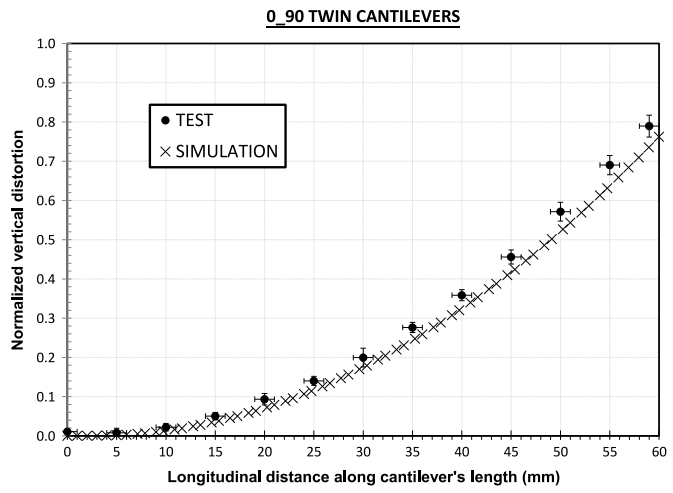

(e) 45_90 twin-cantilever.

Fig. 16. Comparison of experimental and numerical results for distortion after the iterative inherent strain determination process.

\subsection{Validation of results}

The validation of the calculated inherent strains as well as the methodology explained in Section 4.2.1 was carried out observing the behaviour of the remaining twin-cantilevers which present a scanning rotation of 0_90 and 45_90 (see Fig. 4). Both scanning strategies are periodic so that Eq. (8) was used to calculate the normalised inherent strain tensor to be applied in the numerical model:

$$
\boldsymbol{\varepsilon}_{\text {periodic }}^{\text {olnh }}=\frac{\varepsilon_{l}+\varepsilon_{t}}{2}\left\{\begin{array}{l}
1 \\
1 \\
0
\end{array}\right\}=-0.67\left\{\begin{array}{l}
1 \\
1 \\
0
\end{array}\right\}_{x y z}
$$




\section{ARTICLE IN PRESS}
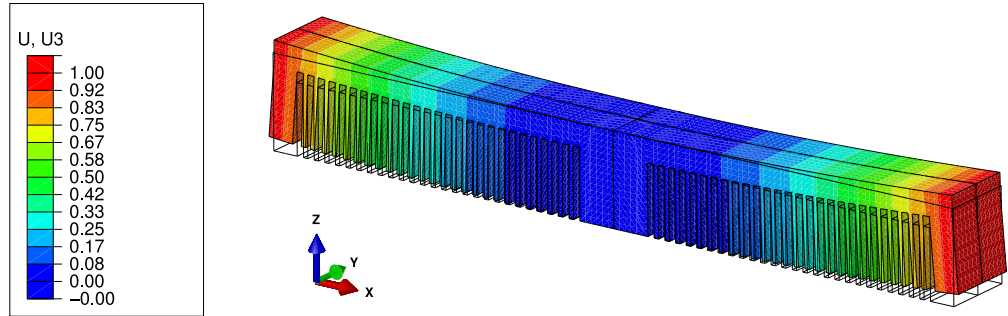

(a) Longitudinal stripes twin-cantilever.
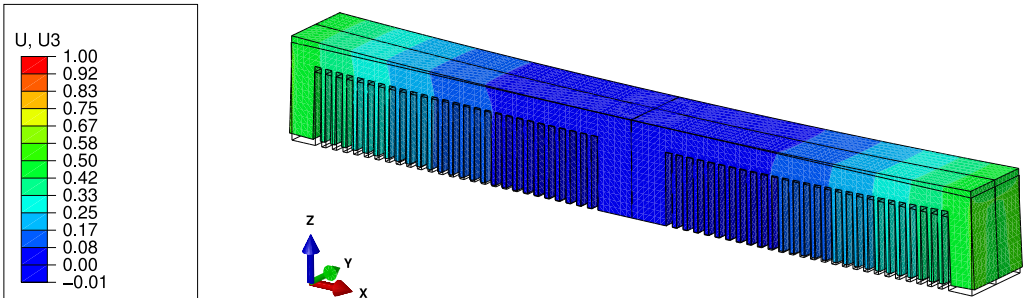

(b) Transversal stripes twin-cantilever.
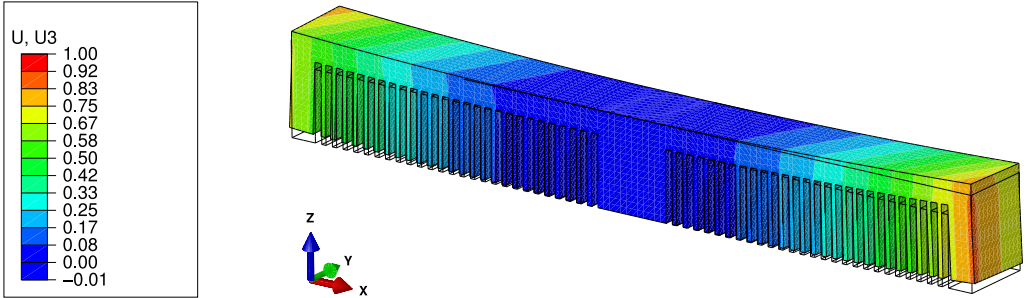

(c) $45^{\circ}$ stripes twin-cantilever.
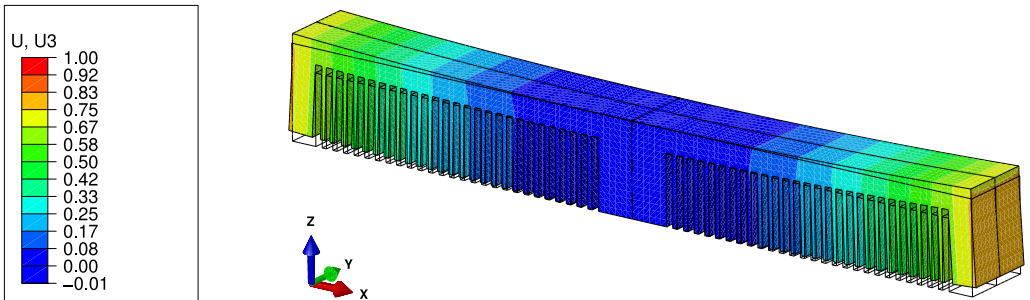

(d) 0_90 twin-cantilever.
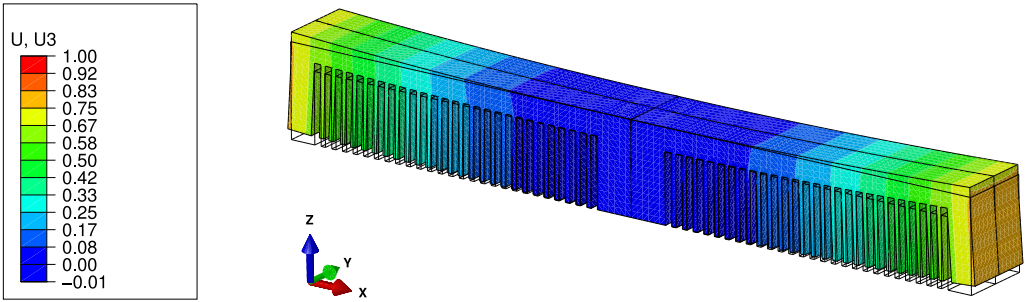

(e) 45_90 twin-cantilever.

Fig. 17. Predicted normalised vertical distortion field (scaled $x 2$ ) after twin-cantilevers were cut-down.

The comparison between numerical and experimental results in terms of vertical distortion of the top surface can be observed in Figs. 16(d) and 16(e). The FEM results match the experimental measurements quite well since the largest difference is less than $10 \%$. The vertical distortion of the deformed structures in Figs. 17(d) and 17(e) is also consistent with experimental results. 


\section{Conclusions}

An empirical methodology to determine characteristic inherent strains for a given scanning strategy is detailed. This result is used as input data for a linear-elastic analyses in order to obtain distortion and residual stress field caused by LPBF processes. Moreover, when the scanning strategy is periodic, the equivalent inherent strains can be assumed as in-plane isotropic, simplifying the implementation of the numerical model.

The development and validation of the methodology have been carried out by manufacturing twin-cantilever coupons following different scanning strategies. Thus, suitability of this methodology is proved at coupon level.

The following practical conclusions can be drawn:

- The distortion is clearly influenced by the chosen scanning strategy and the geometry of the structure. For the twincantilever geometry, longitudinal pattern leads to the largest distortions whereas the transversal pattern to the lowest. Finally, $45^{\circ}$ pattern and $90^{\circ}$ rotating stripes are in-between.

- Inherent strain tensor can be assumed as orthogonal. This result should be checked for other more complex geometries.

- Periodic scanning strategies lead to in-plane isotropic inherent strains.

\section{Acknowledgements}

This work forms part of the DISTRACTION project, supported by the European Union's Horizon 2020 research and innovation programme under Grant Agreement No. 686808. The first author gratefully acknowledges collaboration with Delft University of Technology, specially Dirk Munro.

\section{References}

[1] I. Gibson, D. Rosen, B. Stucker, Additive manufacturing technologies, Rapid Manufacturing Association, Springer Berlin Heidelberg, 2013, pp. 10-12. http://dx.doi.org/10.1520/F2792-12A.2. http://www.ciri.org.nz/nzrma/technologies.html.

[2] T. DebRoy, H. Wei, J. Zuback, T. Mukherjee, J. Elmer, J. Milewski, A. Beese, A. Wilson-Heid, A. De, W. Zhang, Additive manufacturing of metallic components process, structure and properties, Prog. Mater. Sci. 92 (Suppl. C) (2018) 112-224. http://dx.doi.org/10.1016/j.pmatsci.2017.10.001.

[3] J. Kruth, L. Froyen, J.V. Vaerenbergh, P. Mercelis, M. Rombouts, B. Lauwers, Selective laser melting of iron-based powder, J. Mater Process. Technol. 149 (1) (2004) 616-622, 14th Interntaional Symposium on Electromachining (ISEM XIV), http://dx.doi.org/10.1016/j.jmatprotec.2003.11.051.

[4] B. Schoinochoritis, D. Chantzis, K. Salonitis, Simulation of metallic powder bed additive manufacturing processes with the finite element method: A critical review, Proc. Inst. Mech. Eng. B (2015). http://dx.doi.org/10.1177/0954405414567522.

[5] P. Michaleris, Minimization of Welding Distortion and Buckling, Woodhead Publishing Limited, 2011.

[6] J.A. Goldak, M. Akhlaghi, Computational Welding Mechanics, Springer Science \& Business Media, 2006.

[7] L.-E. Lindgren, Computational Welding Mechanics, Elsevier, 2014.

[8] M. Chiumenti, M. Cervera, N. Dialami, B. Wu, L. Jinwei, C.A. de Saracibar, Numerical modeling of the electron beam welding and its experimental validation, Finite Elem. Anal. Des. 121 (2016) 118-133. http://dx.doi.org/10.1016/j.finel.2016.07.003.

[9] L. Papadakis, A. Loizou, J. Risse, J. Schrage, Numerical computation of component shape distortion manufactured by selective laser melting, Proc. CIRP 18 (2014) 90-95. http://dx.doi.org/10.1016/j.procir.2014.06.113.

[10] E.R. Denlinger, M. Gouge, J. Irwin, P. Michaleris, Thermomechanical model development and in situ experimental validation of the Laser Powder-Bed Fusion process, Addit. Manuf. 16 (2017) 73-80. http://dx.doi.org/10.1016/j.addma.2017.05.001.

[11] L.-E. Lindgren, A. Lundbck, M. Fisk, R. Pederson, J. Andersson, Simulation of additive manufacturing using coupled constitutive and microstructure models, Addit. Manuf. (2016). http://dx.doi.org/10.1016/j.addma.2016.05.005.

[12] M. Chiumenti, E. Neiva, E. Salsi, M. Cervera, S. Badia, J. Moya, Z. Chen, C. Lee, C. Davies, Numerical modelling and experimental validation in selective laser melting, Addit. Manuf. 18 (Suppl. C) (2017) 171-185. http://dx.doi.org/10.1016/j.addma.2017.09.002.

[13] C. Li, J. Liu, Y. Guo, Prediction of residual stress and part distortion in selective laser melting, Proc. CIRP 45 (2016) 171-174. http://dx.doi.org/10.1016/ j.procir.2016.02.058.

[14] D. Pal, B. Stucker, A New and Efficient Multi-Scale Simulation Architecture for the Prediction of Performance Metrics of Parts Fabricated Using Additive Manufacturing, TMS2015, 2015.

[15] M. Chiumenti, M. Cervera, A. Salmi, C.A. de Saracibar, N. Dialami, K. Matsui, Finite element modeling of multi-pass welding and shaped metal deposition processes, Comput. Methods Appl. Mech. Engrg. 199 (37)(2010) 2343-2359. http://dx.doi.org/10.1016/j.cma.2010.02.018.

[16] M. Chiumenti, X. Lin, M. Cervera, W. Lei, Y. Zheng, W. Huang, Numerical simulation and experimental calibration of Additive Manufacturing by blown powder technology. Part I: thermal analysis, Rapid Prototyping J. 23 (2) (2017) null. http://dx.doi.org/10.1108/RPJ-10-2015-0136.

[17] T.A. Krol, S. Westhäuser, M.F. Zäh, J. Schilp, G. Groth, Development of a simulation-based process chain strategy for different levels of detail for the preprocessing definitions, 21 (2011) 135-140. http://dx.doi.org/10.11128/sne.21.tn.10081.

[18] N. Keller, F. Neugebauer, H. Xu, V. Ploshikhin, Thermo-mechanical simulation of additive layer manufacturing of titanium aerospace structures, 2013, https://www.researchgate.net/profile/Nils_Keller/publication/275330526_Thermo-mechanical_Simulation_of_Additive_Layer_Manufacturing _of_Titanium_Aerospace_structures/links/5537781d0cf2058efdeabf14/Thermo-mechanical-Simulation-of-Additive-Layer-Manufacturing-of-Titaniu m-Aerospace-structures.pdf.

[19] N. Keller, V. Ploshikhin, New method for fast predictions of residual stress and distortion of am parts, 2015. URL https://sffsymposium.engr.utexas. edu/sites/default/files/2014-096-Keller.pdf.

[20] M. Gouge, P. Michaleris, Thermo-Mechanical Modeling of Additive Manufacturing, Elsevier Science, 2017 URL https://books.google.es/books?id= MuxGDgAAQBAJ.

[21] P. Alvarez, J. Ezenarro, I. Setien, M.S. Sebastian, A. Echeverria, L. Eciolaza, Computationally efficient distortion prediction in powder bed fusion additive manufacturing, in: International Journal of Engineering Research \& Science (IJOER), 2016, ISSN: [2395-6992] [Vol-2, Issue-10, October.

[22] N. Patil, A Novel Numerical Framework for Simulation of Multiscale Spatio-Temporally Non-Linear Systems in Additive Manufacturing Processes (Ph.D. thesis), 2014. http://dx.doi.org/10.18297/etd/1099. 
[23] E. Denlinger, Thermo-Mechanical Model Development and Experimental Validation for Metallic Parts in Additive Manufacturing (Ph.D. thesis), The Pennsylvania State University, 2015.

[24] C. Tsai, W. Cheng, H. Lee, Modeling strategy for control of welding-induced distortion, 1995.

[25] Y. Ueda, H. Murakawa, K. Nakacho, N. Ma, Establishment of computational welding mechanics, Weld. Surf. Reviews(UK) 8 (1) (1997) 265-299.

[26] H. Murakawa, Y. Luo, Y. Ueda, Inherent strain as an interface between computational welding mechanics and its industrial application, Book Inst. Mater. 695 (1998) 597-619.

[27] Y. Ueda, H. Murakawa, N. Ma, Welding Deformation and Residual Stress Prevention, Elsevier, 2012.

[28] J. Wang, S. Rashed, H. Murakawa, Mechanism investigation of welding induced buckling using inherent deformation method, Thin-Walled Struct. 80 (2014) 103-119.

[29] W.E. King, A.T. Anderson, R.M. Ferencz, N.E. Hodge, C. Kamath, S. a. Khairallah, Modeling and simulation of additive manufacturing processes, America (2008) $1-2$.

[30] C. Li, J. Liu, X. Fang, Y. Guo, Efficient predictive model of part distortion and residual stress in selective laser melting, Addit. Manuf. 17 (Suppl. C) (2017) 157-168. http://dx.doi.org/10.1016/j.addma.2017.08.014.

[31] R.M. Jones, Mechanics of Composite Materials, CRC press, 1998.

[32] E.J. Barbero, Introduction to Composite Materials Design, CRC press, 2010. 